\title{
Corela
}

Cognition, représentation, langage

HS-30 | 2020

Phonétique, littérature et enseignement du FLE : théories et recherches

\section{Allitérations, assonances et figuralismes : de leur histoire à leur utilisation en phonétique du FLE}

\author{
Claire Pillot-Loiseau et Claudia Schweitzer
}

\section{OpenEdition}

Journals

Édition électronique

URL : http://journals.openedition.org/corela/10134

DOI : 10.4000/corela.10134

ISSN : 1638-573X

Éditeur

Cercle linguistique du Centre et de l'Ouest - CerLICO

Référence électronique

Claire Pillot-Loiseau et Claudia Schweitzer, «Allitérations, assonances et figuralismes : de leur histoire à leur utilisation en phonétique du FLE », Corela [En ligne], HS-30 | 2020, mis en ligne le 21 mai 2020, consulté le 22 mai 2020. URL : http://journals.openedition.org/corela/10134; DOI : https://doi.org/ 10.4000/corela.10134

Ce document a été généré automatiquement le 22 mai 2020.

\section{(i) (2)

Corela - cognition, représentation, langage est mis à disposition selon les termes de la licence Creative Commons Attribution - Pas d'Utilisation Commerciale - Partage dans les Mêmes Conditions 4.0 International. 


\title{
Allitérations, assonances et figuralismes : de leur histoire à leur utilisation en phonétique du FLE
}

\author{
Claire Pillot-Loiseau et Claudia Schweitzer
}

\section{Introduction}

1 Apprendre une langue étrangère est une tâche à plusieurs niveaux : ainsi que l'affirme Lauret (2007), le lexique renvoie à la mémorisation, la grammaire à l'organisation des mots et, outre les connaissances plutôt formelles comme l'assimilation du vocabulaire et celle des règles grammaticales, la prononciation relève d'un travail spécifique dans la mesure où elle renvoie à une performance physique (Lauret, 2007) d'une part, et qu'elle nécessite des compétences de production et de perception d'autre part. Elle nécessite aussi de cerner la correspondance graphème-phonème, particulièrement complexe en français (Sprenger-Charolles, 2008). Dès le début de la grammaire française au $\mathrm{xVI}^{\mathrm{e}}$ siècle, le lien entre les lettres écrites et leur réalisation correcte est une des préoccupations des grammairiens ${ }^{1}$. Puis, en écho avec ce que décrit également Galazzi (2002) dans son ouvrage, Guimbretière (1996:45) affirme que "c'est avec la mise au point de l'alphabet phonétique international et des débats qu'il a suscité autour des années 1880-1890, que la phonétique est réellement entrée dans l'enseignement et y a tenu depuis une place plus ou moins importante ».

2 Aujourd'hui encore, la prononciation peut faire l'objet d'un apprentissage délicat, car elle joue sur la « sensibilité » non seulement de l'appareil phonatoire, mais aussi celle du système auditif entier (depuis l'oreille externe jusqu'aux aires du traitement du langage) et de la capacité d'auto-évaluation de l'élève. Les apprenants perçoivent et produisent les sons d'une langue étrangère de façon "biaisée » par leur langue maternelle : la primauté de la perception sur la production, après la théorie du crible phonologique (Troubetzkoy, 1970 [1939]), donnera lieu à la publication de différents modèles théoriques d'acquisition d'une langue étrangère (Perceptual Assimilation Model: 
Best, 1994 ; Best et Tyler, 2007 ; Feature Competition Model : Hancin-Bhatt, 1994 ; Speech Learning Model : Flege, 1995 ; Perceptual Magnet Effect Model : Kuhl et al., 2008 ; Phonological Interference Model: Brown, 2000; Ontogeny Phylogeny Model: Major, 2001; Second Language Linguistic Perception Model : Escudero, 2005 ; Automatic Selective Perception Model : Strange, 2011). Certains de ces modèles (Flege, 1995) affirment qu'il est plus difficile de percevoir et donc de produire un segment de la langue étrangère phonologiquement similaire à celui de la langue maternelle (mais proche acoustiquement), plutôt qu'un son entièrement nouveau. Toutefois, la Théorie de la Perception pour le Contrôle de l'Action (Perception-for-Action-Control Theory, Schwartz et al., 2012) se positionne en faveur d'une perception sensorimotrice multimodale, c'est-à-dire: perception auditive, proprioceptive, et du geste articulatoire du segment considéré. Cette perception multimodale permettrait alors au sujet de réajuster rapidement son articulation de ce segment.

3 L'ensemble des difficultés de prononciation d'une langue étrangère nécessitent de la part des apprenants une ouverture vers de nouvelles sonorités (Lauret, 2007) ce qui peut en particulier être permis par l'exploitation du patrimoine littéraire et musical français, pour les raisons suivantes: premièrement, la langue sonne, voire elle peut "chanter", et cela dans sa totalité comme dans chacun de ses segments. Elle est musicale, grâce à ses qualités rythmiques, mélodiques et harmoniques, ce que l'on appelle ses paramètres prosodiques. Deuxièmement, la langue peut peindre ce dont elle parle. Il s'agit de sa fonction mimétique, l'imitation ou la représentation de la nature et, dans un sens plus large ${ }^{2}$, des passions ${ }^{3}$. Une des maintes possibilités pour rythmer et pour colorer un texte ou discours est le principe de la répétition : l'utilisation de rimes, d'assonances, d'allitérations et de différentes figures de style ${ }^{4}$, peuvent engendrer le plaisir de l'oreille, à condition bien évidemment que les répétitions ne soient pas trop stéréotypées. Une autre possibilité est l'introduction de figures suggérant très concrètement une image ou un bruit par l'utilisation de certaines sonorités ou formules évocatrices comme le sont les figuralismes musicaux.

4 L'idée de combiner deux types de travail, l'un axé sur la diction parlée proprement dite, mais à la base d'un texte littéraire, et l'autre fondé sur des extraits musicaux, nous semble favorable pour trois raisons. D'abord, elle sensibilise l'apprenant à la musicalité, et donc aux sonorités de la langue française par un moyen très simple : la musique même (pour l'exemple du coréen : Hee Park, 2002). En effet, Bastian (2000) et Patel (2008) ont montré les similitudes entre le traitement des informations linguistiques et musicales par le cerveau humain.

5 Puis, le chant peut faciliter l'assimilation de la prononciation d'un son précis, car la diction y est en général plus lente que dans la parole parlée. Le travail sur les assonances et allitérations chantées peut donc attirer l'attention de l'apprenant sur la particularité des sons répétés. Il a d'ailleurs été démontré qu'à travers l'écoute globale et le "repérage des répétitions des éléments sonores ", l'organisation poétique d'une poésie parlée ou chantée "guide l'écoute et facilite la compréhension ainsi que la mémorisation » (Arleo, 1997 : 64). Sur un plan plus intuitif encore, le fait de chanter en langue étrangère peut aider l'étudiant à surmonter ses blocages auto-protecteurs (Antier, 2006) et donc l'ouvrir à la découverte sensorielle des sonorités de la langue étrangère.

6 Enfin, le travail musical sensibilise, affine et forme les capacités de perception auditive. D'abord, il mène l'élève à une réception et à un traitement plus intenses et plus 
différenciés des informations perçues (Klemm, 1987; Bastian, 2000). En outre, il favorise notamment les connexions neuronales par l'activation et la création de liens entre les deux hémisphères (Behne, 1995 ; Schlaug et al., 1995 ; Bastian, 2000 ; Patel, 2008). Selon notre approche ici socioconstructiviste, dans une visée communicative, nous considérons que l'apprenant, déjà enrichi par ses expériences sonores et musicales antérieures, se concentre non seulement sur la "reproduction» du son langagier, jugé perceptivement acceptable par un locuteur natif, mais aussi sur une figure musicale, grâce à laquelle il peut développer une image plus riche, car enrichie d'une émotion.

7 De nombreuses études ont montré l'avantage d'impressions pluridimensionnelles pour une mémorisation optimisée. En particulier, Patel (2011), par son modèle OPERA, affirme que la formation musicale améliore le codage neuronal de la parole si cinq fonctions sont remplies $\mathbf{0}:$ Overlap (chevauchement anatomique des réseaux cérébraux qui traitent une caractéristique acoustique utilisée à la fois en musique et parole); P : Précision (la musique impose des exigences plus élevées sur ces réseaux partagés que ne le fait la parole, en termes de précision de traitement) ; E : Émotion (les activités musicales se livrant à ce réseau suscitent une forte émotion positive); $\mathbf{R}$ : Répétition (ces activités musicales sont souvent répétées); A : Attention (ces activités musicales sont associées à une attention ciblée). Il semble bien que le travail sur des textes chantés réunisse ces conditions.

8 Le but de cet article est de proposer des méthodes pour mettre sonorités et images contenues dans un texte ou discours au profit de l'optimisation de la prononciation des apprenants de Français Langue Étrangère (FLE) dans un contexte de plaisir musical. En effet, à notre connaissance, l'immense majorité des recherches sur ce thème concernent des apprenants d'Anglais Langue Étrangère et peu d'investigations ont été conduites concernant les atouts des assonances, allitérations et figuralismes musicaux pour le Français Langue Étrangère. Pour cela, nous présentons d'abord les liens historiques entre parole et chant et les figures de l'assonance, de l'allitération et du figuralisme mises en jeu pour l'expressivité de la langue, avant de détailler avec l'exemple de quelques textes et chansons, des possibilités pour leur utilisation en classe de langue.

\section{Parole, chant, expressivité et poétique}

Poésie et chant visent dès l'Antiquité (et probablement depuis plus longtemps encore) l'émotion. Ainsi, nous connaissons l'image légendaire d'Orphée, poète et chanteur mythique. Les effets positifs de la musique sont aussi évoqués dans la Bible dans l'histoire de David qui guérit par son chant le roi Saül de sa mélancolie. Dès le début de l'histoire que nous connaissons, parole et chant entretiennent les liens les plus étroits et un texte écrit suppose une réalisation sonante, c'est-à-dire orale.

\section{1. La musicalité de la langue}

10 Une fois qu'un texte écrit est devenu sonore, on s'aperçoit facilement des points communs qui le lient si étroitement à la musique. Mélodie, rythme, intonation, accentuation : les paramètres dits prosodiques deviennent sensibles (Firth, 1948; Frost et Guy, 2016; Schweitzer et Dodane, 2016) et transmettent ce que l'on appelle la 
«musicalité » de la langue. Elles font partie de la langue-culture qu'un apprenant en FLE souhaite apprendre et comprendre (Aubin, 2008; Aytekin, 2011). Ces paramètres parlent directement au corps humain avant d'être traité par l'esprit (Aubin, 2008). Rappelons dans ce contexte le concept du double décodage de Fónagy $(1971 ; 1983)$, touchant l'encodage comme le décodage d'un message, et cela en deux démarches consécutives concernant d'abord le signal phonique (signifiants et signifiés arbitraires) et ensuite leur dimension expressive (paralinguistique).

11 Bien évidemment, c'est le texte de départ qui permet une réalisation qu'un auditeur va qualifier comme plus ou moins «musicale ». Les auteurs classiques comme La Fontaine ou Racine montrent par leur sens de la mise en valeur des sonorités et de l'élégance structurelle du français une musicalité évidente dans leurs vers dont la meilleure preuve est la facilité avec laquelle enfants et adultes les mémorisent. Au XIX ${ }^{e}$ siècle, Paul Verlaine déclare dans son poème "Art poétique " (Jadis et Naguère, 1885): «De la musique avant toute chose ", clair manifeste pour l'importance que Verlaine accorde à la musique du vers.

On touche ici à ce que l'école de Jakobson (1963) appelle la «fonction poétique du langage »: le message est centré sur lui-même et sa forme esthétique. Il accentue le caractère matériel des signes linguistiques destinés non seulement à transmettre un message concret, mais aussi à rendre le message visible et sensible. Dans les œuvres poétiques, le son l'emporte alors souvent sur le sens et le lecteur glisse facilement dans un monde imaginaire.

\section{2. Une histoire commune}

Les premières sources musicales et langagières qui nous sont parvenues se trouvent en Chine, environ dix siècles avant Jésus-Christ : elles témoignent du lien étroit entre le langage, la poésie et la danse. Dans l'Antiquité grecque, la musique vocale est également toujours liée à la poésie et occupe une place importante dans l'éducation. Au Moyen Âge, avec le chant grégorien et la poésie des troubadours, les textes chantés rythment les journées et instruisent le peuple (Keil, 2014).

On parle ici d'une musique monodique, c'est-à-dire d'une musique pour une voix seule, sans ou avec accompagnement (on parlera, dans le deuxième cas, d'une "monodie accompagnée »). Les liens entre texte et musique sont soumis à plusieurs changements au cours de l'histoire. Au centre se trouve toujours la question si la musique est prioritaire sur la parole ("prima la musica») comme c'est le cas pour la musique polyphonique de la Renaissance ${ }^{5}$, ou si la parole l'est sur la musique (" prima le parole »). Le deuxième cas, typique notamment pour la musique baroque, et généralement caractérisé par la recherche d'une expressivité extrême par la déclamation du texte chanté qui, pour sa part, doit fournir dans l'idéal au compositeur un matériau sonore riche et évocateur. Voici un champ vaste pour la traduction d'un matériau sonore langagier en matériau sonore musical (Harnoncourt, 1984). Stefano Gervasoni (2010), compositeur contemporain, constate encore la grande importance du statut artistique du texte dans une composition vocale : il crée une « osmose » avec la musique qui vise à créer un horizon de sens. 


\section{3. Le chant dans l'éducation}

15 L'utilité de la musique dans l'apprentissage est connue depuis longtemps. Il suffit de penser à Martin Luther (1844 [1520]) et la grande importance que le réformateur accorde à la musique dans l'éducation, ou à la triade tête-cœur-mains de Heinrich Pestalozzi (1989; $c f$. Lyon, 2014) pour qui la musique, et le chant en particulier, jouent un rôle pédagogique important.

16 Pourtant, l'intégration d'éléments musicaux comme le chant dans les cours de langue n'est pas évidente. Comme le note Aubin (2005), elle dépend avant tout de la personne de l'enseignant, de sa «sensibilité auditive » (ibid.:1) et " gestuelle» (ibid.: 2 ), et de son «inspiration à un moment donné » (ibid.: 2). Aux XVIII et $\mathrm{XIX}^{\mathrm{e}}$ siècles, les maitres de français étaient encore censés enseigner l'art de parler français. Aubin (2005:2) note que «jamais les enseignements du français langue vivante étrangère et de la musique n'auront été explicitement si proches » qu’à cette époque. Ils sont liés par «le plaisir de l'écoute et de la conversation » (ibid. : 2 ).

17 Nous illustrons ces propos d'Aubin par un exemple: traditionnellement, l'enseignement de la prononciation du français repose sur l'apprentissage d'une réalisation optimisée des différents sons/phonèmes. Une méthode pour l'apprentissage de la lecture proposé en 1830 par un certain L. Gavoy à Paris (1830) va plus loin. Le livre intitulé L'Art d'apprendre à lire en chantant commence avec l'indication au maître de laisser chanter les voyelles $a, e, i, o, u$ plusieurs fois pour bien les mémoriser. Ensuite, ces voyelles sont chantées avec différentes consonnes initiales. Sans autre explication méthodologique, l'auteur exprime néanmoins sa conviction ferme que l'apprentissage de la lecture sera plus facile à l'aide du chant. D'autres exemples, comme la "grammaire musicale» de De Vallange (1730), destinée à enseigner le latin par le chant, sont cités par Puren (1988: 29).

Par ailleurs, outre le chant, le jeu avec des associations et un langage figuratif étaient autrefois répandus dans le travail sur la langue. Si la méthode traditionnelle repose sur la répétition de ce que le maître vient de montrer (Puren, 1988), les anciens auteurs profitaient également de ce qui caractérise jusqu'à aujourd'hui l'enseignement musical : le travail avec des images. On trouve alors souvent dans les grammaires et manuels de langue des comparaisons et explications imagées comme celle que Dangeau (1694) donne pour les voyelles nasales : « le nez a quelque part à leur prononciation : \& si vous voulez l'examiner soigneusement, vous verrez que quand vous les prononcez, il se fait quelque petit mouvement dans votre nez " (Dangeau 1694, $1^{\text {re }}$ lettre:7-8). Le langage imaginatif mobilise, outre le savoir articulatoire (l'implication des résonances nasales), tout un spectre d'associations (le mouvement, comme un chatouillement dans le nez, par exemple).

19 Ces approches, à quelques exceptions près abandonnées au cours du XIX ${ }^{e}$ siècle, nous semblent largement intéressantes et justifient de par leur longue tradition notre proposition pour leur ré-utilisation en classe de FLE aujourd'hui.

\section{4. L'expressivité du discours parlé et chanté}

Les jeux sur les sonorités de la langue dans l'objectif d'ajouter à l'expressivité d'un texte ont une longue histoire. Nous nous concentrons notamment ici sur trois phénomènes, dont deux littéraires et un musical: dans la poésie, on relève depuis 
l'Antiquité l'utilisation d'assonances et, un peu plus tard, d'allitérations à des fins suggestives. Il s'agit soit de préciser en quelque sorte le sens général d'un texte par l'utilisation de sons d'un caractère évocateur, soit d'inspirer une ambiance favorable à la compréhension intuitive des mots. Autrement dit, il s'agit de peindre par les sonorités utilisées le contenu du message.

21 L'utilisation des éléments poétiques musicaux de la langue dans l'enseignement de FLE a également une histoire qui remonte au moins au xIx siècle: Galazzi (2002:67) souligne que plusieurs ouvrages phonétiques du début du vingtième siècle, après une première partie indiquant des notions de physiologie et de phonétique expérimentale, fournissaient des textes en orthographe et en phonétique mettant en valeur une certaine musicalité de la langue comme les allitérations et assonances suivantes : «Le mur murant Paris rend Paris murmurant » ou " Non, il n'est rien que Nanine n'honore ${ }^{6}$ ». Galazzi (2002: 103) cite également l'ouvrage de Nyrop (Manuel phonétique du français parlé, paru à Copenhague en 1893 et traduit en français en 1902 par Philipot aux éditions Picard) "excellent représentant de la méthode physiologique tout court " (ibid.:103) : cet ouvrage comprend d'abord des planches anatomiques permettant à l'apprenant de connaitre l'exact fonctionnement articulatoire de chaque phonème du français, puis « des exercices phonétique méthodiques et surveillés qui mènent l'élève à maitriser les organes de la parole tout en développant 'la souplesse du gosier et la finesse de l'ouïe'» (ibid.:103); il se compose ensuite des virelangues tels que le très connu " ton thé t'a-t-il ôté ta toux ?", virelangues également présents (ibid. : 107) dans l'ouvrage de Zünd-Burguet (1902: Méthode pratique, physiologie et comparée de prononciation française, aux éditions Kündig/Elwert, Paris-Marburg-Genève). Ce dernier manuel comprend aussi des fables de La Fontaine en orthographe et en phonétique, tout comme la Petite Phonétique comparée (1912) de Paul Passy (ibid. : 152).

Les musiciens peuvent bien sûr reprendre ces phénomènes et, mettant le texte en musique, les souligner. Ils ont pourtant développé au cours des siècles un autre procédé, combinable avec l'utilisation d'assonances et d'allitérations, qui consiste à peindre également l'idée du texte, qu'elle soit naturelle, affective ou symbolique, mais cette fois avec des moyens musicaux : il s'agit du figuralisme, dont nous allons reparler ultérieurement.

\section{4. 1. L'assonance}

Nous désignons comme assonance, selon la distinction habituellement effectuée (Jarrety, 2001; Bergez et al., 2010, entre autres), la répétition d'un même timbre vocalique dans l'objectif de créer une harmonie sonore. Le dictionnaire de Furetière (1690) constate alors une forte proximité avec le domaine musical: "Quelques-uns se servent de ce mot en Musique pour signifier Consonance. Ce mot vient d'assonare, s'accorder en son ». Selon Jarrety (2001), deux cas différents sont envisageables.

Le premier type est ancien: son usage dans la versification française est même antérieur à la rime. Il s'agit de la répétition de la même voyelle tonique en position finale (accentuée) d'un vers, rimé ou non, et indépendamment des consonnes qui suivent éventuellement. Le deuxième type d'assonance se distingue par la proximité sensible d'un même phonème vocalique à l'intérieur d'un vers ou d'une unité syntaxique. Nous le trouvons par exemple dans l'assonance [i], ici souligné, dans «Le vierge, le vivace et le bel aujourd'hui /Va-t-inl nous déchirrer avec un coup d'aile ịvre» de 
Mallarmé (dans le poème du même nom, 1887), où le texte se voit fortement rythmé par le fait que les assonances sont dans les deux vers placées sur les mêmes positions.

\section{4. 2. L'allitération}

L'allitération concerne la répétition d'un ou de plusieurs sons consonantiques en position initiale, intérieure ou finale de plusieurs mots proches dans un vers ou dans une phrase (p.ex. Fontanier, 1977; Dupriez, 1984). Elle souligne un effet soit harmonique, soit rythmique, soit structurel d'un texte. Fontanier (1977) parle d'une " harmonie imitative» qui "peut servir à marquer le caractère de la pensée, à lui donner plus de trait et de saillie». Deux exemples bien connus sont d'abord la répétition de la liquide [1] figurant la fluidité, et des autres sonantes $[\mathrm{m}]$, [n] et [R], créant un effet harmonieux dans les vers "Sous le pont Mirrabeau coule la Seine / Et ños amours / Faut-il qu'il m'en souvienne " du poème "Le pont Mirabeau » de Guillaume Apollinaire (1912). Beaucoup plus menaçant est l'effet de celle en [s] dans le vers «Pour qui sont ces serpens ${ }^{7}$ qui sifflent sur vos têtes? » de Jean Racine (Andromaque, acte $\mathrm{V}$, scène $5,1667)$, imitant le sifflement du serpent.

\section{4. 3. Le figuralisme}

Un cas particulier constitue le figuralisme musical, une sorte de peinture en musique des images concrètes et/ou émotives (Keil, 2014). Pratique déjà connue au Moyen Âge, le figuralisme se développe notamment à partir du XVI e siècle dans ce que l'on appelle le stile rappresentativo. Nous citons ici la définition du terme de Marc Honegger (1976: 374) qui nous semble assez complète et évocatrice :

Par ce terme [figuralisme] on entend couramment, de nos jours, l'utilisation dans la composition musicale de groupes de notes ou figures dont la disposition et l'effet suggestif sont destinés à évoquer chez l'interprète et l'auditeur une image, un mouvement, un sentiment ou une idée exprimée par le texte qui est mis en musique ou qui inspire celle-ci. À ce titre, le figuralisme est un des moyens de l'expression musicale [...].

27 Le figuralisme peut être suggestif sur plusieurs niveaux, notamment par :

28 - l'allusion à un mouvement comme le fait de monter un escalier, symbolisé en musique par la figure appelé anabasis, anabase, ascensus ou ascensio (Kircher, 1650 ; Mattheson, 1739), c'est-à-dire une montée mélodique. Le figuralisme est certes sensible sur le plan auditif, mais aussi visuel $\left(\right.$ Bartel, 1997) ${ }^{8}$;

29 - le rappel de phénomènes de la nature comme un lieu calme et rassurant ou, au contraire, le bruit d'une tempête. Le figuralisme est descriptif et joue sur la reconnaissance par assimilation à des phénomènes connus: le chant d'un oiseau (comme le motif connu du «coucou»), le bruit du tonnerre, le murmure d'une petite rivière ${ }^{9}$;

30 - l'évocation des sentiments. On parle alors d'un figuralisme affectif. Il peut se manifester par exemple par la figure de la pathopoeia ou pathopoia (Burmeister, 1601 ; cf. Bartel, 1997) qui exprime des passions comme la douleur, la terreur ou la crainte (le terme vient du grec pathos, la souffrance, et poiein, faire, donc: ce qui fait souffrir), symbolisé souvent par des notes chromatiques avec un effet un peu étrange $\left.{ }^{10}\right)$.

31 - l'allusion symbolique, un figuralisme qui impose alors une large connaissance auprès de l'interprète et de l'auditeur, comparable au symbolisme romantique que l'on trouve 
dans la littérature ou dans la peinture. Nous nous permettons de transposer alors le terme de figuralisme dans le domaine de l'image sonore d'une scène ou émotion dans les textes littéraires.

Ce qui nous semble intéressant pour le travail avec des apprenants de FLE musiciens ou non, est le lien possible entre une image musicale, une sonorité précise et une idée extramusicale que nous proposons d'utiliser pour le travail en classe de langue. Les assonances et les allitérations, véritables « figures de diction " (Jarrety, 2001), peuvent jouer un rôle pertinent pour créer un figuralisme.

Il est à noter que Guimbretière (1996:45) propose d'associer un son à une image pour " faire naître chez l'apprenant [de FLE] une représentation mentale du son. Le son devient alors une entité en elle-même qui a une vie propre et qui pourrait avoir une couleur, une odeur, une forme ou une texture éventuellement, tout dépendra du mode de perception que peut avoir l'individu». Dans leur manuel, Kaneman-Pougatch et Pedoya-Guimbretière (1989-2003) illustrent ce concept, par exemple pour l'entité sonore [ui] : à côté de l'image d'une tulipe s'ouvrant progressivement, les auteures expliquent: "pour produire ce son complexe [...] son d'abord labial puis écarté, imaginez une fleur, une tulipe plus précisément qui s'ouvrirait rapidement. Suivez son mouvement : replié comme une fleur à l'ombre, vous vous ouvrez au contact du soleil » (Kaneman-Pougatch et Pedoya-Guimbretière, 1989-2003 : 74).

Nous allons développer dans la partie suivante l'adaptation pédagogique du patrimoine littéraire mais aussi musical, mais allons auparavant rappeler pourquoi l'utilisation de la littérature pour l'apprentissage de la langue étrangère est un atout. Nous sommes conscients des limites d'une telle proposition. La réalisation dépend, comme noté plus haut, beaucoup de la personne de l'enseignant, de sa formation et de sa sensibilité artistique. La même remarque est également valable pour la littérature et la musique choisies. Nous soulignons alors qu'il s'agit d'exemples plutôt "classiques" et en quelque sorte intemporels. Ils veulent motiver l'enseignant de s'inspirer dans ce matériau proposé pour trouver de sa part des exemples techniquement comparables, mais précisément adaptés à ses apprenants : leur âge, leur civilisation, leur culture, leurs intérêts particuliers...

\section{Adaptation pédagogique}

\section{1. Atouts de la littérature comme matériel pédagogique : contre les idées reçues}

35 Nous pourrions au préalable considérer que l'enseignant doit être spécifiquement formé à la connaissance de certains aspects relatifs à la littérature et à la musique pour pouvoir profiter de ces patrimoines littéraire et musical. En outre, ce patrimoine devra être choisi à bon escient pour éviter la création de contextes défavorables à la réalisation de certains phonèmes. Enfin, n'oublions pas que s'adapter au goût des apprenants en matière de littérature et de chant peut au préalable demander une nécessaire phase de découverte de ce patrimoine spécifique, pour éviter que les matériaux sélectionnés sélectionnent un public trop spécifique.

Il convient cependant de nous attarder sur l'importance de lutter contre les idées reçues à propos de l'utilisation de la littérature comme matériel pédagogique : selon 
Savvidou (2004:2), « les raisons pour lesquelles les professeurs considèrent souvent la littérature comme inappropriée à la classe de langue [...] reflètent la séparation historique entre l'étude du langage et celle de la littérature, ce qui a conduit au rôle limité de la littérature en classe de langue ${ }^{11}$ ». Certains textes littéraires ne partagent pas toutes les conventions et règles qui gouvernent les productions verbales standard d'une part, et demandent un plus grand effort dans leur interprétation car leur signification peut être détachée du contexte social immédiat du lecteur d'autre part. Cependant, les frontières entre les discours littéraire et non littéraire ne sont pas si distinctes (Savvidou, 2004). D'autres limites de ce matériel souvent soulignées sont : (i) qu'il s'éloigne de l'authentique et du naturel ${ }^{12}$; qu'il existe un meilleur matériel à utiliser, plus pratique et vocalisé sur le sens plus que la forme (Cook, 1997), et (ii) que le langage y est souvent plus difficile qu'au quotidien (Cariboni Killander, 2011).

Pourtant, le recours à la littérature peut permettre à l'apprenant non seulement de renforcer ses connaissances du langage dans tous ses types discursifs, mais aussi de saisir (i) des nuances de langue plus fines, ainsi que (ii) la créativité et la versatilité qui caractérisent certaines de nos formules verbales, comme par exemple les formes humoristiques (Savvidou, 2004). De plus, grâce à ce matériel, cet apprenant développe aussi, en plus de ses habiletés linguistiques, la pensée critique, la capacité de comprendre d'autres cultures et d'imaginer d'autres modes de vie, mais aussi d'enrichir sa vie émotionnelle et même de développer son attitude empathique, particulièrement en ce qui concerne la poésie chez l'adulte (Cariboni Killander, 2011) ou chez l'enfant (Aydin et Atmaca, 2013). Enfin, comme pour d'autres matériaux, l'avantage d'utiliser la littérature pour la prononciation est de proposer, en y insérant des virelangues et d'autres objets musicaux, du matériel pédagogique en parole continue et non pas en mots isolés, ce qui favorise particulièrement l'amélioration du rythme et de la fluence, mais aussi de la diction et de la qualité vocale dans la prononciation d'un apprenant d'une langue étrangère (Katchen, 1988).

\section{2. Atouts des allitérations et assonances pour l'amélioration des capacités linguistiques}

Nous venons de résumer les principaux atouts de la littérature pour l'apprentissage de la langue étrangère, et plus précisément de sa prononciation. Les recherches précédentes ont également montré que certaines composantes de ce matériel littéraire, en l'occurrence les allitérations et les assonances, pouvaient optimiser les capacités linguistiques d'un apprenant.

\section{2. 1. Mémorisation et compréhension}

39 Lindstromberg et Boers (2008a) ont demandé à six participants belges flamands de 20 et 21 ans de niveau d'anglais langue étrangère B2 de trier en deux catégories (moitié allitérantes à l'initiale de chaque mot/moitié non allitérantes) vingt-six phrases anglaises avec deux mots monosyllabiques. Immédiatement après, les participants ont été invités à écrire autant de phrases qu'ils le pouvaient. En plus de ce test de rappel immédiat, ils ont été soumis à un test de reconnaissance deux semaines plus tard. Lors de ce test différé, les participants ont reçu une liste de cinquante-deux phrases de deux mots comprenant les vingt-six phrases rencontrées dans la tâche de tri et vingt-six autres « distracteurs », dont treize allitérés et treize non. Les auteurs ont demandé aux 
étudiants de cocher les phrases qu'ils se rappelaient de la tâche de tri. Les résultats ont montré que l'ajout d'allitérations révélait un potentiel mnémotechnique considérable qui pourrait être exploité pour faciliter l'apprentissage par groupes de mots, mais aussi que les apprenants ne sont pas très susceptibles de débloquer ce potentiel de manière autonome. En outre, Webb et al. (2013 : 112), Lindstromberg et Boers (2008a ; 2008b) ont constaté que la sensibilisation de l'apprenant à la présence d'assonances ou d'allitérations (Boers et Lindstromberg, 2005) rendait les collocations plus mémorisables que les collocations sans motif, lorsque ces expressions sont considérées comme des éléments décontextualisés, mais également quand ces phrases sont rencontrées dans des textes lus en mettant principalement l'accent sur leur contenu, une semaine après l'apprentissage des expressions contenant des allitérations (Boers et al., 2014).

\section{2. 2. Pouvoir rhétorique de la répétition}

Les assonances et allitérations sont bien un processus de répétition d'éléments du discours. Selon Johnstone (1987), la répétition est un dispositif cohésif du discours : il existe une multitude de types de "réitérations " cohérentes, allant de la répétition exacte de mots ou de phrases à l'utilisation de pronoms cataphoriques et anaphoriques. La répétition a aussi des effets sémantiques. Cependant, cette auteure évoque aussi l'analyse de la répétition comme un dispositif rhétorique ce qui nous concerne davantage dans cette étude: ici, répéter n'est pas juste imiter un modèle pour se conditionner dans une prononciation améliorée : les figures rhétoriques que sont en particulier les assonances et les allitérations rendent le son du discours élégant, créant là une "présence rhétorique " (Johnstone, $1987: 208$ ), c'est-à-dire le premier plan linguistique d'une idée qui peut servir à la rendre persuasive, même sans support logique. Ces figures sont particulièrement employées dans les discours de certains hommes politiques et certaines publicités.

\section{2. 3. Prononciation}

41 L'étude de Thomson (2013) examine dans quelle mesure les professeurs de langue anglaise sont capables d'évaluer de manière critique les idées préconçues et les pratiques d'enseignement dans le domaine de l'enseignement de la prononciation. Pour répondre à cette question, un sondage en ligne a été mené auprès de cinquante-huit enseignants, leur demandant d'indiquer dans quelle mesure ils étaient d'accord ou non avec les déclarations extraites de sites Web et de vidéos YouTube appartenant à des programmes de prononciation, de réduction de l'accent et de modification de l'accent. Thomson $(2013: 227)$ a ainsi mis en évidence, que, pour $77 \%$ de ces enseignants interrogés, notamment l'allitération et les virelangues pouvaient être utilisés comme exercices pour améliorer la prononciation. Selon Ventura (2008), les virelangues, pouvant jouer sur les allitérations et assonances, aident l'apprenant à « délaisser la langue» (Ventura, 2008:9); ainsi, ces étudiants peuvent "saisir dans toute son ampleur la difficulté de langue et, à la fois, [de] jouer avec elle» (ibid.: 9). Ce matériel est cependant souvent réduit à ces virelangues, d'où l'intérêt d'enrichir ce contenu pédagogique par des extraits authentiques de littérature avec ces allitérations et assonances. 
Puisque nous incluons des productions musicales, notamment chantées, dans notre arsenal pédagogique, il convient d'abord d'établir une synthèse relative au rôle du chant et de la musique en Français Langue Étrangère de nos jours.

\section{3. Apports spécifiques du chant et de la musique pour l'apprentissage de la prononciation d'une langue étrangère : synthèse}

Bon nombre de productions chantées possèdent des allitérations et assonances, en plus des rimes. En outre, comme déjà évoqué, les productions musicales, instrumentales ou vocales, peuvent aussi s'enrichir de figuralismes dont nous avons déjà décrit le principe. Si «la compréhension et la mémorisation du vocabulaire et des structures grammaticales, la cohésion de groupe, l'engagement du corps et la sollicitation des deux hémisphères cérébraux » (Arleo, $1997: 63$ ) sont facilités par la chanson, quels sont les principaux atouts des productions musicales pour l'apprentissage de la prononciation du Français Langue Etrangère, par rapport aux productions parlées?

\section{3. 1. Sur le plan suprasegmental}

L'association la plus évidente qui nous vient entre musique, chanson et prononciation, est l'influence de ces productions musicales sur la composante prosodique de la prononciation, à savoir la perception et la production des rythmes, accentuations, et intonations de la langue. En effet, musique et langage partagent des paramètres et fonctions communs dans leur composition (fréquence, intensité, durée ; communication esthétique, structure profonde et de surface). Par exemple, Kolinsky et al. (2009) ont cherché à savoir si la formation musicale facilitait le traitement de l'accentuation lexicale chez les natifs d'une langue sans accentuation lexicale. De jeunes francophones de Belgique peu connaisseurs du flamand et de l'anglais, dont vingt-huit entraînés musicalement (seize années de pratique musicale en moyenne) et trente-deux non musiciens, se sont vu confier deux tâches : une classification accélérée de paires minimales de logatomes qui les obligeait à se concentrer sur un contraste segmental et à ignorer les variations d'accentuation non pertinentes, et la répétition de telles paires impliquant des contrastes segmentaux ou de d'accentuation. Dans ce dernier cas, ces francophones sont généralement "sourds " à l'accentuation lexicale, mais ce fut moins le cas pour les musiciens, ce qui démontre que le savoir-faire musical améliore la sensibilité aux contrastes d'accentuation.

\section{3. 2. Sur le plan segmental}

Les allitérations et assonances concernant davantage cet aspect que l'élément prosodique : il est important de développer les atouts de la chanson et de la musique pour l'acquisition des consonnes et voyelles.

Dans l'étude de Cornaz et al. (2010), une méthode phonétique traditionnelle audio-orale a été légèrement modifiée pour introduire des tâches de voix chantée (vocalises, comptines avec les voyelles antérieures arrondies du français). Huit locuteurs natifs d'italien ont été divisés en deux groupes: l'un pour l'enseignement phonétique commun et l'autre pour l'enseignement phonétique comprenant des tâches de chant. Les sujets ont reçu à l'Université de Turin, entre cinq et neuf heures de formation 
phonétique à la perception et à la production des phonèmes $/ \mathrm{y} /$ et $/ \varnothing /$, à raison de deux séances de deux heures par mois pendant deux mois et d'une heure le jour de la dernière évaluation le troisième mois. Les résultats montrent que les sujets à qui l'on enseigne des tâches supplémentaires chantent et apprennent plus vite que les autres, produisent mieux que l'autre groupe les phonèmes français antérieurs arrondis /y/ et / $\varnothing /$ dans les régions acoustiques attendues pour ces voyelles en français.

Si elle comporte bon nombre de répétitions, un lexique facile à appréhender par l'apprenant, des groupes rythmiques courts, une position privilégiée des phonèmes cibles en position accentuée des points de vue linguistique mais aussi musical (valeur longue, mélodie élevée), des phonèmes cibles dans des contextes segmentaux favorisants (Callamand, 1981) ainsi qu'une mélodie conjointe facilement chantable dans le médium, la chanson dans ces éléments constitutifs optimisera la prononciation des sons étudiés, tout en favorisant motivation, émotion positive (Claerr et Gargan, 1984), mémorisation, voire jeu chez nos apprenants aussi bien enfants qu'adultes. Ajoutons que des activités concernant la voix chantée doivent compléter l'utilisation de la chanson en classe de langue : le travail corporel, vocal (ouverture de la gorge, travail sur les résonances, conscientisation et maîtrise du souffle, travail articulatoire approfondi) et auditif, est indispensable à une production articulatoire détendue, agréable donc et optimisée de la chanson, laquelle n'est de fait qu'une partie de ce travail en voix chantée (Cornaz et Fonio, 2014; Cornaz et Caussade, 2014). Enfin, la musique instrumentale permet un meilleur traitement phonétique des voyelles du Français Langue Étrangère (Miras, 2013).

48 Après une synthèse historique sur les figures rhétoriques de la littérature que sont les allitérations, les assonances et les figuralismes, propos suivis par une récapitulation de leurs apports actuels dans les productions parlées puis de l'apport de productions musicales instrumentales et chantées pour la prononciation d'une langue étrangère, nous proposons à présent d'illustrer nos propos par l'exposé de plusieurs exemples de textes littéraires dans leurs atouts et leur exploitation afin qu'un apprenant de Français Langue Étrangère puisse mieux prononcer cette langue.

\section{4. Atouts et exploitation de matériaux littéraires et musicaux pour optimiser la prononciation de FLE : exemples}

49 Si l'ouvrage de Filliolet et Vandendriessche (2010), pour des apprenants de niveaux A2 à $B 1$, propose pour chaque son et chaque opposition pertinente des poèmes choisis pour la richesse des phonèmes qu'ils contiennent, il a pour but de compléter un cours de phonétique, mais ne propose pas de matériel musical.

50 En complément de cet ouvrage toutefois fondamental dans l'exploitation didactique de la littérature poétique pour optimiser la prononciation de FLE, nous proposons quelques outils supplémentaires dans ce but, exploitant les allitérations, assonances et figuralismes.

\section{4. 1. Ouverture vers une perception fine de sonorités étrangères et jeux de prononciation}

51 Clément Janequin (vers 1485 - 1558) est un compositeur français de la Renaissance, notamment connu pour ses chansons polyphoniques, dont un grand nombre se 
distingue par une forte imagination descriptive. Dans la chanson «Le chant des Oiseaux » (écrite vers 1529), les strophes sont généralement suivies d'un passage imitant les différents chants des oiseaux (notamment le coucou, le merle, le rossignol) par des onomatopées, développées pour la plupart du texte qui précède. La première strophe est consacrée à l'imitation des oiseaux en général («farirariron fere li ioli »), la deuxième imite notamment un petit sansonnet parisien (" ty ty piti pyti, chou chou chou thy thouy, que dy tu tu di ») le troisième le rossignol (avec un long passage composé entre autre de "frian, ticun, qui la ra, huit, fereli fy, cy ty, trr, tu, qui lara, ticun, coqui, teo, tar, tycun, turry, qui-by, trr, coqui, tar, fouquet, quibi », etc.) et la quatrième le coucou («coucou coucou $»$ ).

S'il peut être difficile de faire produire toutes ces onomatopées aussi rapidement par les apprenants, même de niveau avancé, cet extrait peut d'abord, après explication du contexte général de cette chanson empruntée à la littérature musicale, être écouté plusieurs fois par ces apprenants de FLE, en particulier pour la découverte ludique des sons /y/, / $\mathrm{u} /$ comparés à /i/ et /u/ (figure 1 ).

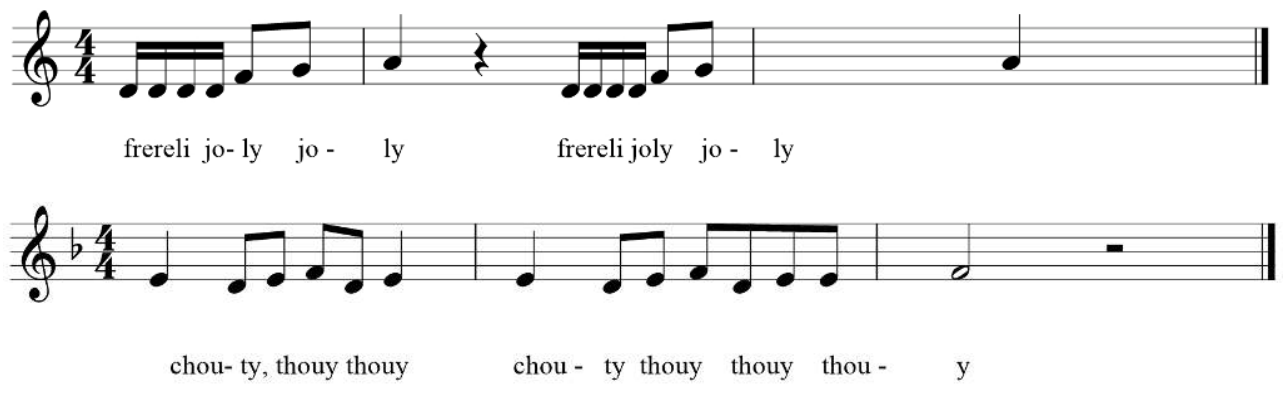

Figure 1 : partition de Janequin « Le Chant des Oiseaux » montrant les onomatopées de chant d'oiseaux

Ces onomatopées pourraient ensuite être reprises à l'isolée afin de constituer des tests perceptifs d'identification des voyelles cibles. Enfin, une tâche d'imitation de ces cris d'oiseaux de l'extrait alors joué au ralenti, en plusieurs groupes d'apprenants, peut clore l'exploitation pédagogique de ce matériel, de manière ludique.

Nos propositions s'inscrivent dans une visée phonostylistique (Léon, 1993), étude des variations phoniques et des effets sonores produits consciemment ou non par le locuteur. En effet, Léon lui-même, argumente la primauté de l'oral sur l'écrit en citant notamment le cas des phénomènes expressifs phoniques qui «s'infiltrent» dans l'écriture artistique (ibid.:30): les jeux phoniques littéraires, poétiques, mais aussi musicaux (ibid. : 140), en font partie.

\section{4. 2. Travail de la diction (Pillot-Loiseau, 2011) et du plaisir de parler}

La chanson «Ce moy de may» («Ce mois de mai ») a été également composée par Clément Janequin en 1529. Le mois de mai, image du printemps, est le moment où l'on plante les arbres. Dans la mythologie, c'est le mois des fêtes où l'on honore la nature, et où on attend le retour des morts. C'est aussi le mois de la déesse Maia (déesse de la fécondité) et de l'amour naissant entre les hommes: le tableau est posé pour cette chanson : il s'agit d'une invitation à l'amour en « ce mois de mai », chanson comportant une allitération avec $/ \mathrm{m} /$ et une assonance avec $/ \varepsilon /$ dont voici le texte du début :

Ce $\underline{\text { mois de }} \underline{\text { Mai, }}$ ce $\underline{\text { mois de }}$ Mai

Ce mois de $\underline{\text { Mai ma verte cotte }}{ }^{13}$ 
Ce $\underline{\text { mois de }} \underline{\text { Mai }} \underline{\text { ma verte cotte }}$

Ce $\underline{\text { mois de }} \underline{\text { Mai, je vestirai }}{ }^{14}$.

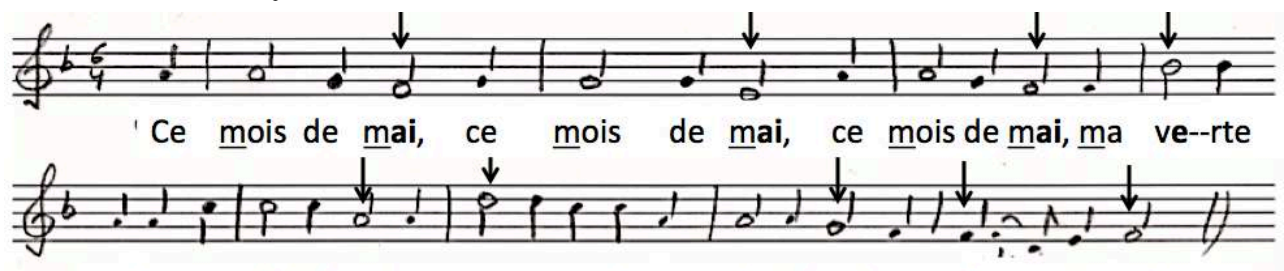

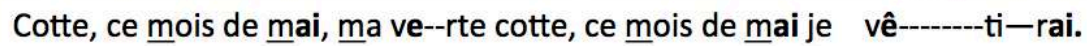

Figure 2: partition de Janequin «Ce moy de may » (« Ce mois de mai ») avec les paroles écrites en français moderne. Les allitérations en / $\mathrm{m} /$ sont soulignées et les assonances en / $\varepsilon /$ sont en gras, toutes situées sur des valeurs musicales longues (flèches), et en fin de mot et de groupe rythmique pour six occurrences sur neuf.

Nous proposons, après écoute et contextualisation de ce chant et l'explication de ses expressions inconnues des apprenants, de chanter ensemble la voix supérieure $\mathrm{du}$ refrain (quatre vers, Figure 2), en prenant du temps pour bien « goûter » la sonorité du $/ \mathrm{m} /$ dont la chanson montre ici une allitération, symbole des plaisirs attendus par les chanteurs. L'apprenant, même débutant, peut chanter en même temps que la voix supérieure, puis en même temps que les quatre voix ensemble. Ensuite, il lui sera possible de répéter chaque partie de cet extrait, avant de pouvoir le produire directement lui-même.

Le rythme, l'intonation et le lexique redondants (Figure 2), les courts groupes rythmiques, la mélodie conjointe, se situant dans le médium (zone de confort) de la voix de tout apprenant, avec un tempo modéré, permettront : 1) l'affinement de la prononciation de la voyelle semi-ouverte $/ \varepsilon /$ en contextes suprasegmentaux favorisants pour six occurrences sur neuf (fin de groupe rythmique, avec en plus une valeur musicale allongée pour toutes les occurrences). Comme le préconise la méthode verbo-tonale (Renard, 1989; Billières, 1995), le contexte segmental favorise la prononciation de cette voyelle au lieu d'une voyelle plus aigue comme /e/ (Callamand, 1981) : dans cette chanson, $/ \varepsilon /$ se trouve toujours précédé d'une consonne grave $/ \mathrm{m} /$, / $\mathrm{v} / \mathrm{ou} / \mathrm{R} /$, et sur une note plus grave pour cinq occurrences sur neuf ; 2) l'appropriation du plaisir de vocaliser chez les apprenants dès le niveau intermédiaire, notamment par la production en initiale de mot des douze occurrences de $/ \mathrm{m} /$ : en effet,

quand on commence à comprendre ce qui se passe vraiment dans sa propre bouche, dans l'acte de parler, quand on contrôle ses muscles buccaux pour prononcer les mots comme il faut, quand on se met véritablement à jouer avec sa langue, ses lèvres, sa mâchoire ou même son souffle, alors parler devient un plaisir - le plaisir de maitriser la technique pour produire des sons plus purs, plus propres, plus beaux. Et les mots deviennent des sortes de friandises qui mettent notre bouche en émoi, en créant des sensations nouvelles sur notre langue et nos lèvres. On se met à prononcer certaines phrases comme on fait rouler un bonbon sur le palais. Il y a, au fond, quelque chose de gourmand dans le fait de parler... Parler pourrait presque se ranger aux côtés des autres plaisirs de la bouche. La prononciation s'apparenterait à une forme de mastication, et rendre plus nette sa diction serait comme savourer un met raffiné. À la manière d'un œnologue se délectant de toutes les nuances d'un grand cru, l'orateur doit apprendre à goûter ses propres mots et phrases, user de toutes les parties de sa langue et de son palais, sentir les vibrations de chaque syllabe, les détacher ou faire les liaisons, contrôler la résonance de sa voix et ses modulations, et déguster les sons ainsi produits.» (site «l'Art de la parole ou la gourmandise des mots », Éloquence magazine, consulté le 5 janvier 20185). 


\section{4. 3. Aide concernant quelques écarts de prononciation spécifiques} exploitation de l'assonance pour améliorer la prononciation de $/ \varepsilon /$, nous présentons quelques exemples de la littérature française accessibles à une exploitation pédagogique, en particulier concernant la prononciation des consonnes françaises.

\section{4. 1. Continuité des fricatives sans affrication}

Il est fréquent que certains apprenants de FLE remplacent les fricatives du français, continues (exemple: [z]), par des consonnes affriquées (exemple :[dz] comme pour des apprenants japonophones car, en japonais, / $\mathrm{z}$ / peut se réaliser comme cette affriquée en position initiale de mot, Kamiyama et al., 2016). «Le chêne et le roseau » (fable écrite par Jean de La Fontaine en 1668) comporte en tout plus de 100 fricatives /f s $\int \mathrm{v} \mathrm{z} \mathrm{3/}$ présentes en contextes segmentaux et suprasegmentaux divers : leur allitération peut également figurer le vent, thème central de la fable :

Le Chêne un jour dit au Roseau :

" $\underline{\text { Vous }}$ avez bien sujet d'accuser la nature;

Un roitelet pour vous est un pesant fardeau ».

Après une mise en contexte, une première écoute-découverte puis l'explication d'expressions non comprises par les apprenants, d'abord sans support écrit, l'enseignant demandera à ses élèves quelles sont les consonnes les plus fréquemment répétées. En outre, la transcription phonétique de certains passages permettra de mettre en relief la présence de liaisons avec / $\mathrm{z} /$ dont l'orthographe ne rend pas compte. Puis, après plusieurs écoutes une fois le support écrit distribué, les apprenants pourront surligner les fricatives dont on aura auparavant expliqué le mode d'articulation, particulièrement son caractère continu. La correction de cet exercice mettra en relief l'abondance de ces consonnes figurant le vent. Selon le niveau des apprenants, il sera possible de lire de façon dialoguée le jeu des deux personnages " chêne " et "roseau ", de répéter les fricatives isolées de certains vers puis ces vers entiers, de répéter chaque vers de cette fable avant d'en lire l'intégrale, etc.

61 Si nous préférons la prose, un extrait de Thérèse Raquin d'Emile Zola (chapitre 1, 1868) pourra aussi être exploité de manière similaire à l'exemple précédent pour le travail des allitérations avec les fricatives, sons suggérant ici l'usure du petit passage décrit dans ce texte.

Enfin, en particulier pour les hispanophones et japonophones de niveau intermédiaire en peine avec la production $\mathrm{du} / \mathrm{v} /$ français, un humoristique extrait - plus proche de nos productions actuelles - de La Leçon d'Eugène Ionesco (écrit en 1950), comportant des allitérations en /f/ (souligné ci-dessous) et /v/ (en gras ci-dessous), sera propice à l'intégration de ces deux fricatives labiodentales :

J'étais tout jeune, encore presque un enfant. Je faisais mon service militaire. J'avais, au régiment, un camarade, vicomte, qui avait un défaut de prononciation assez grave : il ne pouvait pas prononcer la lettre $\underline{\underline{f}}$. Au lieu de $\underline{\mathrm{f}}$, il disait $\underline{\mathrm{f}}$. Ainsi, au lieu de : fontaine, je ne boirai pas de ton eau, il disait : fontaine, je ne boirai pas de ton eau. Il prononçait fille au lieu de fille, Firmin au lieu de Firmin, fayot au lieu de fayot, fichez moi la paix au lieu de fichez moi la paix, fatras au lieu de fatras, fifí, fon, fafa au lieu de fifí, fon, fafa ; Philippe, au lieu de Philippe ; fictoire au lieu de fictoire ; $\underline{f}$ évrier au lieu de février; mars-avril au lieu de mars-avril ; Gérard de Nerval et non pas, comme cela est correct, Gérard de Nerval ; Mirabeau au lieu de Mirabeau, etc., 
au lieu de etc., et ainsi de suite etc. au lieu de etc., et ainsi de suite, etc. Seulement il avait la chance de pouvoir si bien cacher son défaut, grâce à ses chapeaux, que l'on ne s'en apercevait pas.

\section{4. 3. Distinction entre occlusives non voisées et voisées}

63 Le poème «Effet de nuit » de Paul Verlaine, publié dans le recueil Poèmes saturniens de 1866, dessine une image nocturne («nuit, noir ») cauchemardesque (« gibet, pendus, mâture des loups, horreur »), sensible par exemple dans les allitérations des soixanteseize occlusives répétées pour ce sonnet de vers à 10 ou 12 pieds (quatorze /p/ comme dans " épine épars "; treize / $\mathrm{t} /$; onze / $\mathrm{k}$ / comme dans « bec avide des corneilles »; six /b/ ; vingt-six /d/ et six /g/). Il pourra être travaillé comme les exemples précédents, mais son atout est que sa mise en musique a fait l'objet d'une composition instrumentale en 1901 par Sylvi Lazzari, dont la musique est accessible sur Youtube ${ }^{16}$ : celle-ci figure musicalement le paysage nocturne avec une instrumentation basse, un tempo musical et un phrasé longs et lents, avec une dynamique expressive. Par conséquent, avant de découvrir le contenu verbal de ce poème, il pourrait être intéressant d'en présenter le contexte par une écoute puis une évocation libre de ce que le passage instrumental évoque pour les apprenants de FLE. Par la suite, l'enseignant pourra poursuivre le travail de ce texte de manière analogue aux étapes proposées dans la fable "Le chêne et le roseau ", en adaptant bien entendu ses propos concernant les consonnes occlusives pour rendre saillants leur lieu d'articulation et leur voisement.

64 Si l'on préfère un passage en prose pour ce travail sur ces occlusives, leur allitération est également manifeste dans le Passage de Verlaine, extrait de ses ouvrages «Variétés I » et "Variétés II ", écrit par Paul Valéry entre 1924 et 1930: par exemple, les allitérations figurent le bâton de Verlaine décrit dans cet extrait comme frappant le sol quand celui-ci marche dans Paris en boitant. Après un travail analogue à celui des exemples précédents, la prise de conscience de ce figuralisme peut aussi s'effectuer, au final, en incitant des apprenants de FLE de niveau avancé à lire ce passage en voix déclamée avec une accentuation d'insistance sur ces occlusives.

\section{4. 3. 3. Consonnes sonantes / $\mathrm{m} \mathrm{n} /$ et distinction $/ \mathrm{r} \mathrm{l} /$}

65 Le poème «Colloque sentimental », dernier texte du recueil Les Fêtes galantes de Paul Verlaine, de 1869, montre deux personnages fantomatiques dans un parc solitaire. Dans une ambiance de désespoir, il évoque leur passé douloureux et développe un regard nostalgique, voire sombre sur leur relation amoureuse. Le poème entier, et notamment l'extrait suivant, révèle de nombreuses allitérations avec les consonnes $/ 1 \mathrm{~m} \mathrm{n} \mathrm{r/,} \mathrm{mais}$ surtout celles avec / $\mathrm{r} /$, principalement en initiale de mot pour /l/ et en final pour / $\mathrm{r} /$ :

Leurs yeux sont morts et leurs lèvres sont molles,

Et l'on entend à peine leurs paroles.

66 À l'aide d'un travail pédagogique semblable aux exemples précédents, la distinction entre $/ 1 /$ et $/ r /$ pour des apprenants japonophones de niveau intermédiaire pourra être travaillée à l'aide de ce matériel, mais aussi le caractère "sonant » de toutes ces consonnes, dont le /r/ quand il se trouve au voisinage d'un segment voisé. 
67 En outre, ce poème a été mis en musique en 1987 par Léo Ferré ${ }^{17}$ qui joue fortement sur les figuralismes pour rendre compte de l'atmosphère triste, comme gelée qui entoure les deux protagonistes. Ainsi, la mélodie reste toujours calme, déterminée par des petits intervalles mélodieux : les deux personnes semblent être déjà presque mortes. Chacun des deux premières phrases que nous proposons de chanter, commence avec une catabasis, descente mélodique exprimant la faiblesse. Elle est (pour le deuxième et le quatrième vers) suivie d'une anabasis (montée, voir supra). Dans le troisième vers, l'allitération du /l/ soutient l'idée de mollesse et de douceur (donc de nostalgie), le demi-ton qui suit pour introduire la montée mélodique renforce encore cet aspect (Figure 3). La musique décrit alors le vide extérieur et intérieur qui caractérise ce poème.

Là encore, avant tout travail « verbal » sur ce poème, son contexte pourra être présenté par une écoute puis une évocation libre de ce que la chanson évoque pour les apprenants de FLE, même si ceux-ci n'en n'ont pas compris la profondeur ou tout le sens caché.
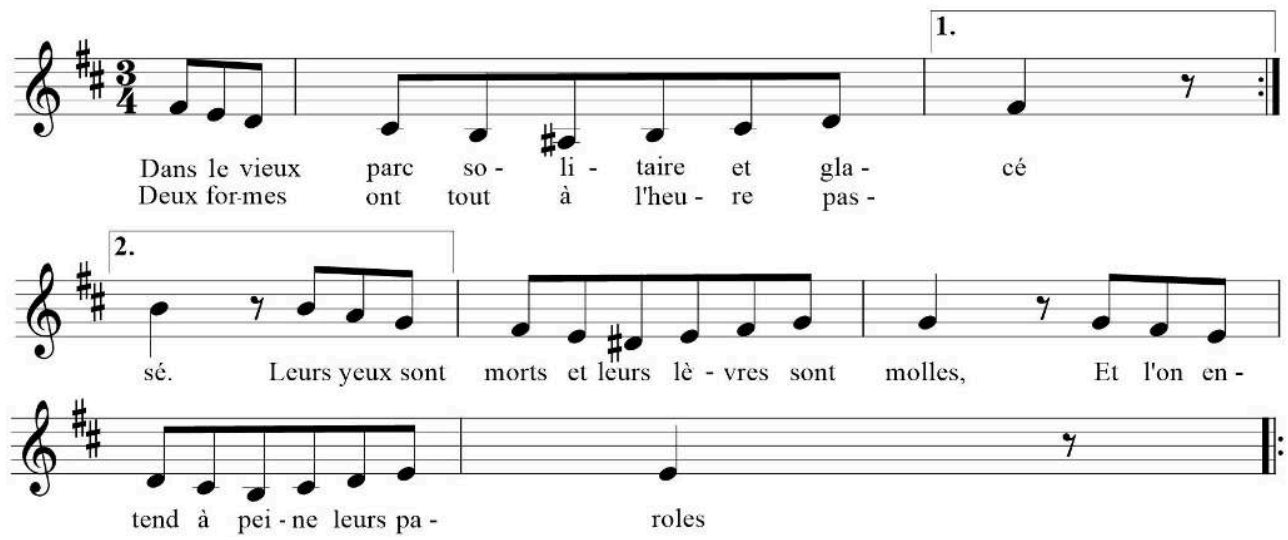

Figure 3 : Partition de la mise en musique du « Colloque sentimental », de Verlaine, chanté par Léo Ferré

\section{4. 4. Figuralismes}

\section{4. 4. Exemples précédents}

La description des quelques exemples précédents a montré la présence de figuralismes «de parole» ou musicaux, et leur possible exploitation pédagogique : nous pouvons effectivement rappeler les onomatopées chantées figurant le "chant des Oiseaux » (Janequin), des fricatives figurant le vent dans « le chêne et le roseau » de La Fontaine, les occlusives allitérées figurant le frappement du bâton de Verlaine boitant dans la rue, l'adaptation instrumentale de "Effets de Nuit» de Verlaine, figurant le triste paysage nocturne, et la mise en chanson du poème "colloque sentimental » de Verlaine.

\section{4. 4. La notion de "figuralisme articulatoire"}

Nous avons rappelé que la littérature connaît les jeux d'allitérations et d'assonances qui créent des effets sonores. Cependant, à part la sonorité même, la forme articulatoire intervient également si nous parlons ou chantons le son plein d'un « a » ou bien le son plus aigu d'un « $i$ ». À partir des travaux de Fónagy (1983), nous pouvons affirmer que 
les voyelles arrondies ou étirées se distinguent par leur couleur (qui les lie avec la mise en son d'une certaine passion) et par leur geste articulatoire sensible, mais aussi visible (qui les lie avec une représentation optique de cette passion). Ainsi, la répétition de voyelles arrondies dans le poème " $Q u^{\prime} A m o u r$ mon cœur, qu'Amour mon âme sonde ${ }^{18}$ " de Ronsard (Les Amours, 1553, poème 21) peut illustrer ce que nous nous permettons d'appeler une "assonance articulatoire»: toutes ces voyelles, certes de sonorités différentes, partagent le point articulatoire commun d'être arrondies. Cet arrondissement labial figure ici la sensualité de l'amour et peut être par conséquent considéré comme un «figuralisme articulatoire » (Figure 4 en haut). Dans le poème « Le Vampire » de Baudelaire (Les Fleurs du Mal, poème 32, 1857), la réitération des voyelles antérieures étirées /i e $\varepsilon$ / figure la perfidie et le danger (Figure 4 en bas). Par l'éventuelle mise en musique du texte, ces caractéristiques articulatoires peuvent être renforcées, ou bien se perdre dans le flux de la mélodie ou des harmonies, ce qui est le cas de la mise en musique de ce dernier poème de Baudelaire par William S.N. ${ }^{19}$ (entre 3:11 et 3:18 en particulier pour «j'ai prié le glaive rapide de conquérir ma liberté »).

\section{"Qu'Amour mon cœur, qu'Amour mon âme sonde »}

\section{"J'ai prié le glaive rapide de Conquérir ma libertés "}

Figure 4

En haut : premier vers du poème de Ronsard Les Amours (1552) : texte avec allitérations en /m/ (soulignés) et « figuralisme articulatoire » de voyelles arrondies (lèvres arrondies au-dessus de ces voyelles).

En bas : deux premiers vers du poème « Le Vampire » de Baudelaire avec « figuralisme articulatoire » de voyelles antérieures étirées (lèvres étirées au-dessus de ces voyelles).

71 L'exploitation pédagogique des «figuralismes articulatoires" nous paraît complémentaire aux atouts des allitérations et assonances, car, en plus d'un travail perceptif et de production se concentrant sur les caractéristiques sonores des segments cibles, le travail des « figuralismes articulatoires » introduit celui du geste articulatoire, comme celui de l'arrondissement labial qui concerne la majorité des voyelles françaises.

\section{Conclusion}

Nous avons exposé dans cet article les racines historiques des allitérations, assonances et figuralismes musicaux, et donné des exemples de l'utilisation de sources littéraires et musicales afin de profiter au mieux des atouts de ces figures de style pour l'amélioration de la prononciation du FLE. Il faut bien entendu souligner que ces phénomènes surviennent dans d'autres ressources que la littérature : cinéma ("Y'a pas d'hélice, hélas! C'est là qu'est l'os », Louis de Funès dans La grande vadrouille), autres chansons ne faisant pas partie du patrimoine que nous avons étudié (comme par exemple "Ta Katie t'a quittée » de Bobby Lapointe ou "j'ai envie de vous » de Elie Semoun pour le figuralisme articulatoire des voyelles arrondies), rap et slam ${ }^{20}$, publicité (Grunig, 1990 ; Martin, 2002 ; exemples : assonance en /a/ : «Carglass répare, 
Carglass remplace »; assonance en /õ/ : « le thon c'est bon, alors, étonnez vos plats ! ), dessin animé (figuralisme musical quand une flûte produit une mélodie chromatique descendante pour figurer la chute de Donald, série Saludos Amigos créée en 1943 par Walt Disney ; Alarcon, 1987).

Enfin, dans notre partie consacrée à l'adaptation pédagogique de ces matériaux phonétiques spécifiques, si nous avons insisté sur l'importance de l'écoute et de l'interprétation de ce patrimoine littéraire et musical, n'oublions pas qu'ils peuvent constituer un point initial à partir duquel il deviendra possible de proposer à nos apprenants d'inventer un pareil texte puis de le produire. Ainsi, un tel travail à partir d'échantillons littéraires et musicaux ne se contentera pas d'une simple reproduction, mais peut aussi s'inventer : l'apprenant peut ainsi s'approprier des critères de style et les utiliser pour l'apprentissage phonétique : ce travail est alors personnel et adapté à chacun.

\section{BIBLIOGRAPHIE}

ALARCON I., 1987, «Figuralisme et écriture musicale dans le dessin animé », Vibrations, $\mathrm{n}^{\circ} 4$, p. $110-127$.

ANTIER E., 2006, « Prononcer la langue en chantant : un travail phonétique au cœur de l'énonciatif (Comptes rendus d'expériences pédagogiques) », Revue japonaise de didactique du français, vol. 1, n 1, p. 155-164.

ARLEO A., 1997, «Grille n 2 . Activité pédagogique. Chanter en cours de langues », Cahiers de l'APLIUT, vol. 17, n 2, p. 63-65.

AUBIN S., 2005, « Maître de langue, professeur de langue et enseignement de la musique du français (XIX siècle) ", Documents pour l'histoire du français langue étrangère ou seconde [En ligne], $\mathrm{n}^{\circ}$ 35, p. 1-13.

-, 2008, « Pourquoi enseigner les musiques de langue-culture? ", Synergies Espagne, n 1, p. 41-46.

AYTEKIN H., 2011, « L'Exploitation de la chanson en classe de langue étrangère », Ondokuz Mayls

Üniversitesi Eğitim Fakültesi Dergisi, seconde [Revue de la faculté d'éducation de l'université

d'Ondokuz Mayıs], vol. 30, nº 1, p. 145-156.

AYDIN E. et ATMACA H., 2013, « La poésie dans l'enseignement de FLE aux enfants », Electronic Turkish Studies, vol. 8, $\mathrm{n}^{\circ} 10$, p. 97-103.

BARTEL D., 1997, Handbuch der musikalischen Figurenlehre [Manuel théorique des figures musicales], Laaber, Laaber.

BASTIAN H. G., 2000, Musik-erziehung und ihre Wirkung [L'éducation musicale et ses effets], Mayence, Schott.

BEHNE K.-E., 1995, « Vom Nutzen der Musik » [De l'utilité de la musique], Musikforum, nº 83, p. 27-39. 
BERGEZ D., GÉRAUD V. et ROBRIEUX J.-J., 2010, Vocabulaire de l'analyse littéraire, Paris, Armand Colin, coll. «Cursus : Lettres ».

BEST C. T., 1994, « The emergence of native-language phonological influences in infants : A perceptual assimilation model ", in J. C. GOODMAN et H. C. NUSBAUM (dir.), The development of speech perception: The transition from speech sounds to spoken words, Cambridge, MIT Press, p. 167-224.

BEST C. T. et TYLER M. D., 2007, « Nonnative and second-language speech perception: Commonalities and complementarities ", in M. J. MUNRO et O.-S. BOHN (dir.), Second Language speech learning: the role of language experience in speech perception and production, Amsterdam, Jonh Benjamins, p. 13-34.

BILLIÈRES M., 1995, « Didactique des langues et phonétique ; la place du verbo-tonal », RPA, $\mathrm{n}^{\circ} 114$, p. 43-64.

BOERS F. et LINDSTROMBERG S., 2005, « Finding ways to make phrase-learning feasible : The mnemonic effect of alliteration », System, vol. 33, $n^{\circ} 2$, p. 225-238.

BOERS F., LINDSTROMBERG S. et WEBB S., 2014, « Further evidence of the comparative memorability of alliterative expressions in second language learning ", RELC Journal, vol. 45, nº 1, p. 85-99.

BROWN C., 2000, « The interrelation between speech perception and phonological acquisition from infant to adult », Second language acquisition and linguistic theory, $\mathrm{n}^{\circ} 1, \mathrm{p} .4-64$.

BURMEISTER J., 1601, Musica autoschédiastiké,Rostock, Reusner.

CARIBONI KILLANDER C., 2011, « Poetry in foreign language teaching. Aspects of a major challenge », in Proceedings of the International Conference of Education, Research and Innovation (ICERI), Madrid, 14-16 november 2011, p. 18-26.

CALLAMAND M., 1981, Méthodologie de l'enseignement de la prononciation : organisation de la matière phonique du français er correction phonétique, Paris, CLE International

CLAERR T. A. et GARGAN R., 1984, « The Role of Songs in the Foreign Language Classroom », OMLTA Journal, p. 28-32.

CоOK G., 1997, « Language play, language learning », ELT journal, vol. 51, n 3, p. 224-231.

CORNAZ S., HENRICH N. et VALLÉE N., 2010, « L'apport d'exercices en voix chantée pour la correction phonétique en langue étrangère : le cas du français langue étrangère appliqué à des apprenants italiens d'âge adulte ", Recherche et pratiques pédagogiques en langues de spécialité - Cahiers de l'Apliut, vol. 29, nº 2, p. 103-119.

CORNAZ S. et CAUSSADE D., 2014, « Musique, voix chantée et apprentissage : une revue de littérature et quelques propositions d'exploitation en didactique de la phonétique des langues ", Revue Électronique du Centre de Recherche sur les Identités Nationales et l'Interculturalité (e-CRINI), $\mathrm{n}^{\circ} 6$, p. 1-34.

CORNAZ S. et FONIO F., 2014, « Présentation et premiers résultats de la conception d'un référentiel de compétences en pratiques artistiques et apprentissage des langues ", Éditions du CRINI, $\mathrm{n}^{\circ} 6$, p. 1-32.

DANGEAU L., 1694, Essais de grammaire en trois lettres, Paris, Coignard.

DUPRIEZ B., 1984, Gradus. Les procédés littéraires, Paris, Domaine français.

ESCUDERO P., 2005, Linguistic Perception and Second Language Acquisition: Explaining the Attainment of Optimal Phonological Categorization, PhD thesis, LOT Dissertation Series 113, Utrecht University. 
FLEGE J. E., 1995, « Second language speech learning: Theory, findings, and problems », in W. STRANGE (dir.), Speech perception and linguistic experience : issues in Cross-Language Research, Timonium, York Press, p. 233-277.

FILLIOLET C. et VANDENDRIESSCHE A., 2010, La phonétique par les textes, Paris, Belin, coll. « FLE ». FIRTH J. R., 1948, « Sounds and Prosodies », Philological Society, vol. 47, nº 1, p. 128-152.

FóNAGY I., 1971, « Double coding in speech », Semiotica, nº 3, p. 189-222.

-, 1983, La vive voix. Essais de psycho-phonétique, Paris, Payot.

FONTANIER P., 1977, Les figures du discours, Paris, Flammarion.

FOURNIER, J.-M., 2007, « La généralité dans les théories du son à l'âge classique », HEL : Histoire Épistémologie Langage, vol. 29, $\mathrm{n}^{\circ}$ 1, p. 85-103.

FROST D. et GUY R., 2016, «L'innovation est le ton qui fait la chanson : une approche musicoprosodique en secteur Lansad », Cahiers de l'APLIUT [en ligne], vol. 35, nº 1, p. 1-20.

FURETIÈRE A., 1690, Dictionnaire universel, contenant généralement tous les mots françois tant vieux que modernes, et les termes de toutes les sciences et des arts, La Haye, A. et R. Leers.

GALAZZI E., 2002, Le son à l'école. Phonétique et enseignement des langues (fin XIX siècle-début XXe siècle), Milano, Editrice La Scuola.

GAVOY L., 1830, L'Art d'apprendre à lire en chantant, ou Nouvelle méthode de lecture en vingt-quatre leçons, suivies du premier livre de "Télémaque », Paris, Roy-Terry.

GERVASONI S., 2010, « Raisons et occasions dans le choix d'un poème qui devient musique ». Disponible sur : <http://www.stefanogervasoni.net/mobile/index.asp?page=writings\&id=9> (consulté le 3 janvier 2018).

GUIMBRETIÈRE É., 1994, Phonétique et enseignement de l'oral, Paris, Didier Hatier.

GRUNIG B., 1990, Les Mots de la Publicité : L'Architecture du Slogan, Paris, Presses du CNRS.

HARNONCOURT N., 1984, Le discours musical, Paris, Gallimard.

HANCIN-BHATT B., 1994, « Segment transfer : A consequence of a dynamic system », Second Language Research, vol. 10, n 3, p. 241-269.

HEE PARK S., 2002, "L'oral et les apprenants coréens. Problèmes de phonétique et de culture », ELA - Études de linguistique appliquée, vol. 126, nº 2, p. 201-210.

HINDRET J., 1696, L'art de prononcer parfaitement la langue françoise (vol. 1), Paris, d'Houry. JAKOBSON R., 1963, «Linguistique et poétique », in Essais de linguistique générale, Paris, Éditions de Minuit.

HONEGGER M., 1976, Dictionnaire de la musique : technique, formes, instruments, Paris, Bordas.

JARRETY M., 2001, Lexique des termes littéraires, Paris, Librairie générale française.

JOHNSTONE B., 1987, « An introduction », Text-Interdisciplinary Journal for the Study of Discourse, vol. 7, $\mathrm{n}^{\circ}$ 3, p. 205-214.

KAMIYAMA T., DETEY S. et KAWAGUCHI Y., 2016, « Les japonophones », in S DETEY, I. RACINE, Y. KAWAGUCHI et EYCHENNE J. (dir.), La prononciation du français dans le monde : du natif à l'apprenant, Paris, CLE International, coll. « Référence », p. 155-161. 
KANEMAN-POUGATCH M. et PEDOYA-GUIMBRETIÈRE É., 1989-2003, Plaisir des sons, enseignement des sons du français, Paris, Hatier-Didier.

KATCHEN J. E., 1988, « Mastering English Pronunciation through Literature », 14th Annual Meeting of the International Conference on Language Teaching / Learning of the Japan Association of Language Teachers, 28 pages.

KEIL W., 2014, Musikgeschichte im Überblick [Aperçu de l'histoire de la musique], Stuttgart, Fink. KIRCHER A., 1650, Musurgia universalis, 2 vol., Rome, Corbelletti et Grignani.

KLEMM G., 1987, Untersuchungen über den Zusammenhang musikalischer und sprachlicher Wahrnehmungsfähigkeiten [Recherches sur les liens entre les compétences de perception musicale et linguistique], Frankfort/Main, Lang.

KOLINSKY R., CUVELIER H., GOETRY V., PERETZ I., et MORAIS J., 2009, « Music training facilitates lexical stress processing ", Music Perception : an Interdisciplinary Journal, vol. 26, n 3, p. 235-246.

KUHL P. K., CONBOY B. T., COFFEY-CORINA S. et al., 2008, « Phonetic learning as a pathway to language : new data and native language magnet theory expanded (NLM-e) », Philosophical Transactions B of the Royal Society, vol. 363, p. 979-1000.

LÉON P. R., 1993, Précis de phonostylistique du français : parole et expressivité, Paris, Nathan Université, coll. « Linguistique ».

LAURET B., 2007, Enseigner la prononciation du français : questions et outils, Paris, Hachette, coll. « $\mathrm{f}$.

LINDSTROMBERG S. et BOERS F., 2008a, « The mnemonic effect of noticing alliteration in lexical chunks ", Applied Linguistics, vol. 29, n 2, p. 200-222.

,$- 2008 \mathrm{~b}$, « Phonemic repetition and the learning of lexical chunks : The power of assonance ", System, vol. 36, $\mathrm{n}^{\circ} 3$, p. 423-436.

LUTHER M., 1844 [1520], Les propos de table (éd. française), Paris, Garnier.

LYON J., 2014, Pestalozzi et la musique, Paris, Publibook.

MAJOR R. C., 2001, Foreign accent : The ontogeny and phylogeny of second language phonology, Mahwah, Lawrence Erlbaum.

MARTIN E., 2002, « Mixing English in French advertising », World Englishes, vol. 21, nº 3, p. 375-402. MATTHESON J., 1739, Der vollkommene Capellmeister [Le parfait maître de chapelle], Hamburg, Herold. MIRAS G., 2013, « "Enseigner/apprendre” la prononciation autrement: une approche psychosociale musique-parole ", Recherches en didactique des langues et des cultures. Les cahiers de l'Acedle, vol. 10, $\mathrm{n}^{\mathrm{o}} 1$, p. 1-29.

PAGANI-NAUDET C. et SCHWEITZER C. (en préparation), « Description de la prosodie par les grammairiens ( $\mathrm{XV}^{\mathrm{e}}$ et $\mathrm{XVI}^{\mathrm{e}}$ siècles) », in C. DODANE et C. SCHWEITZER (dir.), Description de la parole. De l'introspection à l'instrumentation.

PALSGRAVE J., 1530, Lesclarcissement de la langue francoyse, Londres, Pynson et Haukyns.

PATEL A. D., 2008, Music, Language, and the Brain, Oxford, Oxford University Press.

-, 2011, « Why would musical training benefit the neural encoding of speech ? The OPERA hypothesis », Frontiers in Psychology, vol. 2, article 142, p. 1-14. 
PESTALOZZI J. H., 1898, Comment Gertrude instruit ses enfants (4e édition revue et corrigée) / par Pestalozzi (traduction française de E. Darin), Paris, Delagrave.

PILLOT-LOISEAU C., 2011, « Place de la phonétique dans la revue La voix parlée et chantée », Observatoire musical français, série « Conférences \& Séminaires ", n 47, p. 53-71.

PUREN C., 1988, Histoire des méthodologies de l'enseignement des langues, Paris, Nathan-CLE international, coll. « Didactique des Langues Étrangères ».

RENARD R., 1989, La méthode verbo-tonale de correction phonétique, Paris, Didier-Érudition.

SAVVIDOU C., 2004, «An integrated approach to teaching literature in the EFL classroom », The Internet TESL Journal, vol. 10, $\mathrm{n}^{\circ}$ 12, p. 1-6.

SCHLAUG G., JÄNCKE L., HUANG Y., STAIGER J. F. et STEINMETZ H., 1995, « Increased Corpus Size in Musicians ", Neuropsychologica, $n^{\circ}$ 33, p. 1047-1055.

SCHWARTZ J.-L., BASIRAT A., MÉNARD L. et SATO M., 2012, « The Perception for Action Control Theory (PACT) : a perceptuo-motor theory of speech perception ", Journal of Neurolinguistics, vol. 25, $\mathrm{n}^{\circ} 5$, p. 336-354.

SCHWEITZER C. et DODANE C., (2016, July), « De la notation musicale à la transcription de la prosodie : Description de la prosodie du français $\mathrm{du} \mathrm{XVI}^{\mathrm{e}}$ au début du Xx ${ }^{\mathrm{e}}$ siècle ", in SHS Web of Conferences (vol. 27 : Congrès Mondial de Linguistique Française - CMLF 2016), p. 1-17.

SPRENGER-CHAROLLES L., 2008, « Correspondances graphème-phonème et phonème-graphème: une comparaison de l'anglais, du français, de l'allemand et de l'espagnol », in A. DESROCHERS,

F. MARTINEAU et Y.C. MORIN (dir.), Orthographe française : Évolution et pratique, Ottawa, Éditions David, coll. « Voix savantes », p. 213-225.

STRANGE W., 2011, « Automatic selective perception (ASP) of first and second language speech : A working model », Journal of Phonetics, vol. 39, $n^{\circ} 4$, p. 456-466.

THOMSON R. I., 2013, « ESL teachers' beliefs and practices in pronunciation teaching : Confidently right or confidently wrong? ", in J. LEVIS et K. LEVELLE (dir.), Proceedings of the 4th Pronunciation in Second Language Learning and Teaching Conference, Aug. 2012, Ames, Iowa State University, p. 224-233.

TROUBETZKOY N., 1970 [1939], Principes de phonologie, Paris, Klincksieck.

VENTURA D., 2008, « De l'utilité des acrostiches, des tautogrammes et des virelangues en cours de FLE. Enjeux pédagogiques et didactiques », in La culture de l'autre [Recurso electrónico] : l'enseignement des langues à l'Université : Deuxième Rencontre Hispano-français de Chercheurs (SHF/ APFUE), École Normale Supérieure Lettres et Sciences Humaines (26 au 29 novembre 2008) = Segundo Encuentro Hispanofrancés de Investigadores (APFUE/SHF), p. 41. La clé des langues.

WEBB S., NEWTON J., et CHANG A., 2013, « Incidental learning of collocation », Language Learning, vol. $63, \mathrm{n}^{\circ} 1$, p. 91-120.

Références concernant le matériel littéraire et musical évoqué dans la partie « adaptation pédagogique » de cet article ${ }^{21}$

Références littéraires

Poésie :

BAUdelaire C., 1857, « Le Vampire », Les Fleurs du Mal, nº 32.

LA FONTAINE J. DE, 1668, « Le chêne et le roseau », Fables, Livre I, 22. 
RONSARD, PIERRE DE, 1553, «Qu'Amour mon cuoeur, qu'Amour mon ame sonde », Les Amours, nº 21.

VERLAINE P., 1866, « Effet de nuit », Poèmes saturniens.

-, 1869, «Colloque sentimental », Les Fêtes galantes.

Prose :

VALÉRY P., 1921, Passage de Verlaine.

zoLA É., 1867, Thérèse Raquin.

Théâtre :

IONESCO E., 1950, La Leçon.

Références musicales ${ }^{22}$

FERRÉ L., 1987, Colloque sentimental. Enregistrement : <https://www.youtube.com/watch? $\mathrm{v}=4 \mathrm{JQYuylR5} \mathrm{ew}>$.

JANEQUIN C., 1529, Ce Moy de may. Exemple d'enregistrement : <https://www.youtube.com/watch?

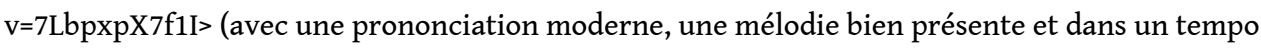
bien accessible pour des amateurs). Partition en libre accès : <http://imslp.org/wiki/ Ce_Moys_de_May_(Janequin\%2C_Cl\%C3\%A9ment) $>$ et $<$ http://www.free-scores.com/ partitions_telecharger.php?partition=2834>.

JANEQUIN C., vers 1529, Le Chant des oiseaux. Exemples d'enregistrements : <https://

www.youtube.com/watch?v=ceS9-RUpzXQ> (dans un tempo accessible pour des amateurs) ou (avec une mise en scène animatrice, mais plus difficile à reproduire $:<h t t p s: / /$ www.youtube.com/watch?v=XoTfSQOqOis>). Un tutoriel pour la mélodie de la voix d'alto se trouve ici : <https://www.youtube.com/watch?v=_7N7CPcLZHs> (pour l'exemple « frere li joli » : vers 1'18). Pour la voix de ténor : <https://www.youtube.com/watch?v=yFDrulEzT9k>avec l'exemple « chouty thouy vers 2'29). Partition en libre accès : <http://imslp.org/wiki/ Le_chant_des_oiseaux_(Janequin\%2C_Cl\%C3\%A9ment)>.

LAZZARI S., 1901, Poèmes saturniens. Exemple d'enregistrement : <https://www.youtube.com/watch? $\mathrm{v}=1 \mathrm{krIjF} 0 \_\mathrm{sNE}>$.

WILLIAM S.N., mise en musique du poème « Le Vampire » de Baudelaire : <https:// www.youtube.com/watch?v=LF5REaqgybs>.

\section{NOTES}

1. Leurs travaux ont soit un objectif normatif, c'est-à-dire l'explication d'une prononciation correcte aux Français, soit un but pédagogique pour l'apprentissage du français par les étrangers, comme la première grammaire française de Palsgrave (1530), écrite pour les Anglais. Voir à ce sujet Fournier (2007) et Pagani-Naudet \& Schweitzer (en préparation).

2. Possible grâce à la codification et la représentation rhétorique et gestuelle des passions, notamment à l'âge classique.

3. Dès l'Antiquité, les philosophes soulignent la parenté des deux arts littéraire et pictural. Aristote les admet tous les deux au rang des sciences " poḯtiques ». Il place pourtant la poésie avant la peinture. L’âge classique a ensuite codifié les passions et leur représentation rhétorique et gestuelle et ainsi permis leur exploitation dans un sens plus large. 
4. Comme l'anaphore et l'épiphore (la répétition d'un même mot soit en position initiale, soit finale d'une proposition) ou l'homéotéleute (la reprise d'une finale ou d'un groupe final dans le principe d'un écho).

5. Dans une musique polyphonique, plusieurs voix ou parties mélodiquement indépendantes sont superposées. La technique d'écriture peut reposer sur les règles du contrepoint.

6. Voltaire, cité dans l'ouvrage de Rousselot et Laclotte Précis de Prononciation Française, paru en 1902 aux éditions Welter.

7. Ce « $\mathrm{s} »$ final se prononçait à l'époque de Racine (voir Hindret J., 1696).

8. Voir infra, la mise en musique du poème « Colloque sentimental » de Verlaine par Ferré (1986).

9. Voir infra, les compositions « Les oiseaux » de Janequin et « Effets de nuit de Lazzari » (1904).

10. Voir infra, Ferré (1986).

11. "The reasons why teachers often regard literature as inappropriate to the language classroom [...] reflect the historic separation between the study of language and the study of literature, which has led to the limited role of literature in the language classroom. »

12. Ces deux termes, selon Cook (1997), sont synonymes et renvoient au langage utilisé dans la communication par des locuteurs natifs adultes ou enfants durant leur acquisition de ce langage. Cet auteur met toutefois en cause cette définition car un langage à la grammaire et aux vocabulaires simplifiés tels qu'on l'utilise avec l'enfant, n'est pas naturel ou authentique pour un apprenant d'une langue étrangère.

13. Le terme cotte, au Moyen Âge un vêtement porté des deux sexes, est à l'époque de Janequin notamment utilisé pour désigner une jupe plissée à la taille, portée par les paysannes et femmes du peuple.

14. "vêtirai " peut aussi bien être écrit en français moderne pour ne pas perturber les apprenants, une fois que le support écrit leur a été distribué.

15. < http://coacheloquence.com/lart-de-la-parole-ou-la-gourmandise-des-mots/>. Voir aussi Dangeau (1694).

16. Par exemple <https://www.youtube.com/watch?v=lkrljF0_sNE> (consulté le 28 mars 2018).

17. Sur son album « On est pas sérieux quand on a 17 ans » : https://www.youtube.com/watch? v=4JQYuylR5ew consulté le 27 mars 2018

18. Orthographe originelle de 1553 : «Qu'Amour mon cuoeur, qu'Amour mon ame sonde ».

19. <https://www.youtube.com/watch?v=LF5REaqgybs> (consulté le 28 mars 2018).

20. Par exemple : «Ça commence par un moment de flottement quand le soleil recule », début de « Nuit », slam de Grand Corps Malade.

21. Nous n'ajoutons pas des références bibliographiques précises pour ces œuvres, accessibles facilement dans de maintes éditions modernes et même sur Internet.

22. Pour les pièces musicales, nous donnons des exemples d'enregistrements, accessibles facilement sur Internet (état 28 mars 2018) et, si possible, des indications pour des partitions en libre accès.

\section{RÉSUMÉS}

Cet article présente d'abord une section historique montrant l'importance des allitérations et assonances en littérature française, mais aussi celle du figuralisme musical. Allitérations et assonances se caractérisent par la répétition de consonnes ou voyelles, propices à toute 
pédagogie de la prononciation: elles améliorent la mémorisation, la compréhension, et la prononciation. Après cette première section, une sélection de plusieurs textes littéraires, dont certains mis en musique, est proposée, pour leur exploitation pédagogique de la prononciation du Français Langue Étrangère : ouverture vers une perception fine de sonorités étrangères et jeux de prononciation, travail de la diction et du plaisir de parler, aide concernant quelques écarts de prononciation spécifiques (continuité des fricatives sans affrication, distinction entre occlusives non voisées et voisées, consonnes sonantes $/ 1 \mathrm{~m} \mathrm{n} \mathrm{r} /$ et distinction entre $/ \mathrm{r} /$ et $/ \mathrm{l} /$ ), «figuralisme articulatoire ».

This article first presents a historical section showing the importance of alliterations and assonances in French literature, but also that of musical figuralism. Alliterations and assonances are characterized by the repetition of consonants or vowels, encouraging to any pedagogical pronunciation activity: they improve memorization, comprehension, and pronunciation. After this first section, a selection of several literary texts, some of which are set to music, is proposed, for their pedagogical use in improving the pronunciation of French as a foreign language: opening towards a fine perception of foreign sounds and pronunciation games, the work of diction and the access to a pleasure of speaking, help concerning some specific pronunciation errors (continuity of fricatives without affricates production, distinction between unvoiced and voiced plosives, sonorous consonants / $1 \mathrm{mnr} /$ and distinction between $/ \mathrm{r} /$ and /l/), "articulatory figuralism".

\section{INDEX}

Keywords : Alliteration, assonance, musical figuralism, literature, pedagogy, pronunciation, French as a Foreign Language

Mots-clés : Allitération, assonance, figuralisme musical, littérature, pédagogie, prononciation, Français Langue Étrangère

\section{AUTEURS}

\section{CLAIRE PILLOT-LOISEAU}

Laboratoire de Phonétique et Phonologie (LPP) UMR 7018, Université Paris 3 Sorbonne Nouvelle, CNRS

\section{CLAUDIA SCHWEITZER}

Histoire des théories linguistiques (HTL) UMR 7597, Université Paris 3 Sorbonne nouvelle, CNRSUniversité Paul Valéry Montpellier 3, Département musicologie, Route de Mende, 34199 Montpellier cedex 5, France 